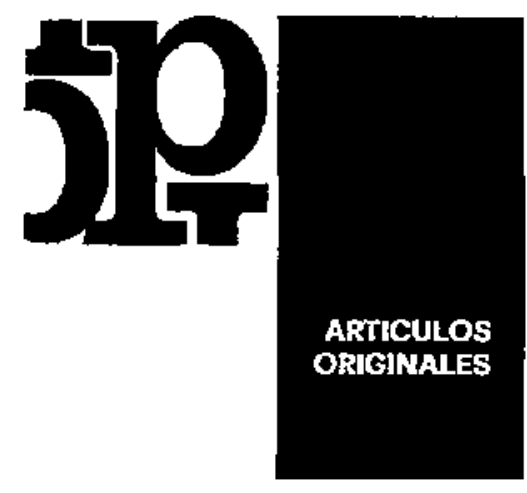

\title{
Efecto de la metoclopramida en la presión de esfínter gastroesofágico en lactantes con reflujo gástrico
}

DRES.: OSVALDO DANUS *, FRANCISCO LARRAIN * y AUGUSTO LARRAIN * .

El reflujo gastroesofágico (RGE) es una condición frecuente en el lactante y que determina síntomas importantes en una alta proporción de ellos. Entre los síntomas destaca el vómito crónico que puede ser causa de desnutrición y los procesos broncopulmonares recidivantes. Con menor frecuencia pueden apreciarse signos precoces de compromiso esofágico por esofagitis péptica.

Aunque en la mayoría de estos pacientes, tanto los síntomas, como el reflujo mismo tienden a atenuarse con el transcurso de los meses, es inportante el número de lactantes que requieren de medidas terapéuticas por la intensidad o la persistencia de los síatomas.

Hasta este momento Ias medidas terapéuticas de orđen médico están dirigidas fundamentalmente a evitar los factores que condicionan el reflujo: tratamiento postural, espesamiento y fraccionamiento de la alimentación. Estas medidas bastan para atenuar el cuadro en la gran mayoría; sin embargo, en algunos niños se hace sentir la necesidad de disponer de otras medidas terapéuticas.

La metoclopramida, un derivado de la procairamida, ba demostrado poseer efectos claros en el vaciamiento gástrico (1) y en la contracción del colon (2). Igualmente parece ser efectiva en la estimulación de la musculatura lisa que constituye el esfínter gastroesofágico. Sin embargo, los resultados obtenidos en este último sentido, todos en adultos, no han sido plenamente concordantes. En efecto, Heitmann y Moller (3) en normales y Larraín y cols. (4) en pacientes con reflujo, aprecian aumento significativo de la presión de esfínter gastroesofágico con el estímulo de metoclopramida endovenosa. Por otra parte Glanville y Walls (5) no aprecian modificaciones del reflujo G. E. radiológico después del uso de la droga.

\footnotetext{
- Gastroenterologia, Departamento de Pediatría. Hospital Roberto del Río.

* Gastroentcrología. HospitaI J. J. Aguirre.
}

Nos ha parecido que es importante precisar el papel que juega la metoclopramida en el lactante, en quien se sabe que el proceso de eficiencia esfinteriana se encuentra en evolucion (6).

Material y MÉTodo. Se procedió a analizar la respuesta manométrica del esfínter gastroesofágico en 11 lactantes portadores de reflujo gastro-esofágico sintomático demostrado radiológicamente. Las principales características clínicas del grupo aparcen en la Tabla No 1 . Seis mujeres y

$$
\text { TA B L A } 1
$$

CARACTERISTICAS CLINICAS EN 11 LACTANTES CON REFLUJO GASTROESOFAGICOS

\begin{tabular}{|c|c|c|c|c|c|}
\hline Caso & Edad & Peso & Peso & Sintomas & $R X$ \\
\hline 1 & $5 \mathrm{~m}$ & 4.880 & 74 & Bronq. obs. & \\
\hline 2 & $17 \mathrm{~m}$ & 11.700 & 102 & Regurgitación & +++ \\
\hline 3 & $6 \mathrm{~m}$. & 5.500 & 61 & $\begin{array}{l}\text { Bronq. obs. } \\
\text { Vómit. crón. } \\
\text { Bronq. obs. }\end{array}$ & +++ \\
\hline 4 & $2 \mathrm{~m}$. & 5.000 & 98 & $\begin{array}{l}\text { Desnutrición } \\
\text { Vómito crón. }\end{array}$ & $\begin{array}{l}t+t \\
+t+\end{array}$ \\
\hline 5 & $9 \mathrm{~m}$. & 6.800 & 78 & Vómito crón. & \\
\hline 6 & $8 \mathrm{~m}$. & 7.100 & 86 & $\begin{array}{l}\text { Desnutrición } \\
\text { Vómito crón. }\end{array}$ & $\begin{array}{l}+++ \\
+++\end{array}$ \\
\hline 7 & $6 \mathrm{~m}$. & 6.250 & 74 & Vómito crón. & \\
\hline 8 & $4 \mathrm{~m}$. & 5.200 & 85 & $\begin{array}{l}\text { Bronquitis obs. } \\
\text { Vómito crón. }\end{array}$ & $\begin{array}{l}+++ \\
++t\end{array}$ \\
\hline 9 & $5 \mathrm{~m}$ & 5.070 & 76 & Vómito crón. & +++ \\
\hline 10 & $8 \mathrm{~m}$ & 6.700 & 82 & $\begin{array}{l}\text { Vómito crón. } \\
\text { Vómito crón. }\end{array}$ & $+t+$ \\
\hline $\mathfrak{1} 1$ & $16 \mathrm{~m}$. & 9.700 & 91 & Desnutrición & +++ \\
\hline
\end{tabular}

5 hombres cuyas edades fluctuaban entre 2 meses y 1 año 5 meses. Ocho de los lactantes presentaban retraso ponderal de acuerdo al criterio de Gómez (7) con peso que oscilaba entre el 74 y $86 \%$ del ideal requerido para la edad considerando el peso de nacimiento. 
El motivo de consulta fue: vómito crónico en 7 , bronquitis obstructiva recidivante en 1 y vó. mito crónico asociado a bronquitis obstructiva en 3. En todos los lactantes el estudio radiológico dirigido demostró reflujo gastro-esofágico espontáneo y repetido. La magnitud del reflujo radiológico fue evaluada de 1 a 3 de acuerdo al criterio adoptado en publicaciones anteriores (6) (3: reflujo espontáneo y repetido; 2 : reflujo que aparece con maniobras especiales, y 1: reflujo provocado por sifonaje de agua).

El estudio manométrico se realizó mediante el sistema de 2 catéteres con orificios distales separados por 3 cms. entre sí, y bajo perfusión continua siguiendo la metódica presentada en publicación anterior (6). Una vez efectuado el registro basal, se fijó la sonda de modo que el orificio distal quedara enclavado en el esfínter gastro-esofágico. Se suministró metoclopramida I. $M$. en dosis de $0,3 \mathrm{mg} / \mathrm{kgr}$. y se procedió a registrar presión esfinteriana y ondas esofágicas inferiores durante 1 minuto a intervalos de 5 minutos y por lapso de 30 minutos.

Resultadoos. La presión basal de esfínter gastroesofágico en el grupo de pacientes varió entre 5 y $12 \mathrm{~mm} \mathrm{Hg}$. con promedio de $6,9 \mathrm{~mm} \mathrm{Hg}$. La diferencia con el promedio encontrado en lactantes normales con similar distribución etaria y estado nutritivo, es significativo. (Figura 1).

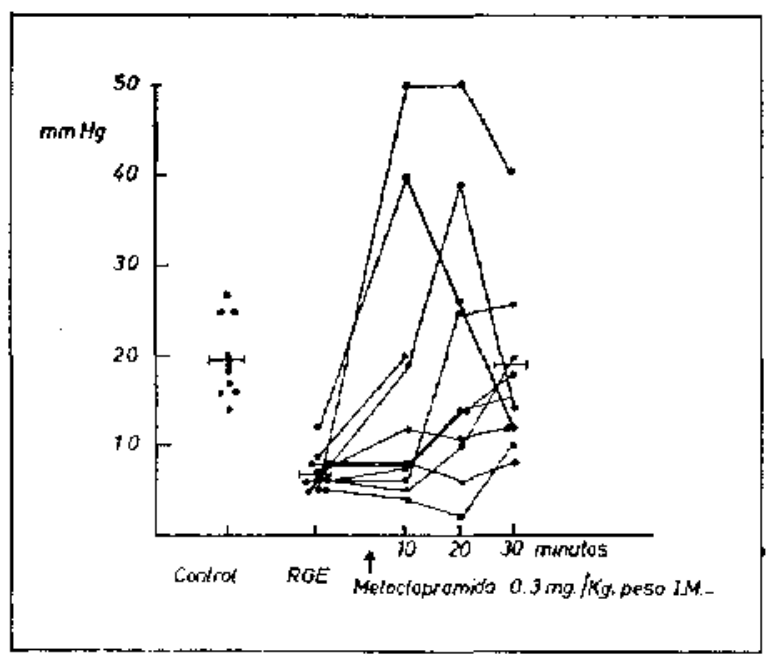

Fig. Ne I

Después del estímulo con metoclopramida intramuscular se apreció respuesta variable en la presión de esfínter y cuyo detalle se presenta en la Tabla 2. En 5 pacientes la respuesta fue inmediata (5') apreciándose elevación de la presión a cifras normales y manteniéndose en estos rangos durante los 30' de la exploración. En otros 4 el efecto aparece más tardíamente $\left(15-20^{\prime}\right)$ pero a los 30' existen presiones sobre $15 \mathrm{~mm} \mathrm{Hg}$. En 1 paciente (caso 2) se obtiene alza de presión sólo
TA B L A 2

PRESION DE ESFINTER GASTROESOFAGICO EN REPOSO Y DESPUES DE ESTIMULO CON METOCLOPRAMIDA INTRAMUSCULAR EN 11 LACTANTES CON REFLUJO GASTROESOFAGICO

Presión esfínter gastroesofigico ( $\mathrm{mm} \mathrm{Hg}$.)

Caso Basal Metoclopramida $(0,3 \mathrm{mg} . \mathrm{Kg}$. I. M.)

\begin{tabular}{rrrrrrrl}
\hline & & 5 & 10 & 15 & 20 & 25 & 30 (minutos) \\
1 & 5,5 & 50 & 50 & 50 & 50 & 50 & 40 \\
2 & 5 & 4 & 4 & 4 & 2 & & 10 \\
3 & 12 & 25 & 40 & 27 & 26 & 26 & 12 \\
4 & 7 & 16 & 12 & 35 & 11 & 12 & \\
5 & 8,5 & 11 & 20 & & & & \\
6 & 8 & 8 & 8 & 8 & 6 & 8 & \\
7 & 6 & 6 & 5 & 6 & 10 & 20 & \\
8 & 8 & 8 & 8 & 14 & 14 & 8 & 15 \\
9 & 6 & 6 & 8 & 24 & 14 & 18 & \\
10 & 5 & & 19 & 25 & 39 & 23 & 14 \\
11 & 6 & 6 & 6 & & & 25 & 26 \\
Promed. 6,9 & & & & & & 19
\end{tabular}

a $\operatorname{los} 30^{\prime}$ (10 $\mathrm{mm} \mathrm{Hg}$.), la que no alcanza rango de normalidad aunque duplica la cifra basal. En otro paciente (caso 6) no se aprecia modificación de la presión de esfínter gastroesofágico en el lapso de $30^{\prime}$. El promedio obtenido a Ios $30^{\prime}$ es de 19 $\mathrm{mmHg}$, similar al obtenido en niños normales.

Comentario. Durante el período de observación no se apreció complicaciones atribuibles al uso de metoclopramida en nioguno de los pacientes.

Los resultados obtenidos de la presión de esfínter gastroesofágico con las dosis señaladas de metoclopramida pureden considerarse bastante satisfactorias ya que en 10 de los 11 lactantes se logró aumentos importantes de la presión basal. En 9 de éstos, las cifras basales ascendieron a rangos de normalidad.

Aunque estos parámetros no traducen directamente la existencia de reflujo gástrico, la suficiencia del esfínter es un elemento fundamental en el mecanismo de contención del estómago y su corrección debe contribuir en grado importante a la normalización del problema.

En el presente trabajo el efecto de la metoclopramida fue medido sólo hasta los 30'. Existiría la duda sobre el beneficio clínico de su uso si el efecto sobre la presión del esfínter no se prolongara mucho más. Sin embargo, creemos que aunque asi fuese, este lapso sería suficiente para beneficiar al paciente debido al tipo de alimentación del lactante, fundamentalmente líquida, y a la aceleración del vaciamiento gástrico inducido por la nietoclopramida.

En nuestra experiencia no se aprecio relación entre presión basal de esfínter y magnitud de la respuesta al estímulo, situación descrita por otros autores. 
Se presentan los resultados obtenidos con el uso de metoclopramida intramuscular en la presión de esfinter gastroesofágico en 11 lactantes con reflujo gástrico sintomático demostrado radiológicamente.

En 9 lactantes se aprecia alza de presión, lográndose valores en rango normal en períodos variables entre 5 y 30 minutos después del estímulo. $A$ los 30 minutos el promedio alcanzado es similar al obtenido en niños normales en condiciones basales. En 1 paciente el aumento de presión fue tardia y no alcanzó a valores normales. En otro no se apreció modificación de la presión basal en el lapso estudiado.

\section{SUMMARY}

The action of the metoclopramide on the rest gastroesophageal sphincter pressure was studied in 11 infants with symptomatic gastro-esophageal reflux.

An increase of the rest pressure to normal range was obtained in 9 infants in 5 to 30 minutes after metoclopramide. One patient showed late an incomplete recovery of the sphincter pressure and in other one, no action on the rest pressure was noticed.
1.- Johnson, $A, G$. The action of metoclopramide on human gastroduodenal motility. Gut 12: 421, 1971.

2.-Eisner, $M$. Gastrointestinal effects of metoclopramide in man. In vitro experiments with human smooth muscle preparations. Brit. Med. J. 4: 679, 1968.

3.- Heitmann, P.; Moller, N. The effect of metoclopramide on the gastroesophageal junctional zone and the distal esophagus in man. Scand. J. Gastroent. S: 621,1970 .

4.--Larrain, A, O.; Uribe, P.; Wunkhaus, R.; Ayala, $M$. Evaluación manonétrica de la acción de la metoclopramida en pacientes portadores de reflujo gastroesofágico. Rev. Med. Chile. 101: 129, 1973.

5.-Glonville, J. N.; Walis, W. D. Effect of intravenous metoclopramide on gastroesophageal reflux. Gut. 13: 31,72 .

6.-Danás, O.; Neira, M.; Larraín, A. Estudio manométrico de esófago en el reflujo gastrocsofágico del lactante. Pediatría, 16: 52, 1973.

7.-Gómez, F.; Ramos Galyán, R.; Cravioto, J.; Freenk, $S$. Malnutrition in infancy and children with special reference to kwashiorkor. Advances in Pediatrics VП: 134, 1955. 